\title{
EVALUATION OF SUGARCANE WAX EXTRACTION CONDITIONS
}

\author{
Michelli Aparecida Bacetti, Patricia Fazzio Martins Martinez.
}

\begin{abstract}
Brazil is typically the world's largest producer of sugarcane. Consequently, the processing of this raw material generates a high amount of by-products, such sugarcane peel. Thus, in this project, sugarcane wax extraction was evaluated through the fractionation of the sugarcane peel using different mass-to-feed ratio, particle size, and extraction time. The trials were done using hexane as solvent in a Soxhlet extractor. Total crude yields were modelled using the software STATISTICA 7.0 and central compound experimental design to gain a better understanding of the conditions required to achieve optimum extraction.
\end{abstract}

\section{Key words:}

Sugarcane peel wax, by-product valuation, process optimization.

\section{Introduction}

With the processing of large volumes of sugarcane, there is a high generation of by-products, such the peel originated from the sugarcane preparation for juice production. Therefore, the investigation of sugarcane wax extraction from this by-product represents one of the opportunities to promote the valuation of these residues, which could even replace commercial waxes such Carnauba and Candelilla.

Natural waxes have applicability in the textile, paper and packaging, food, chemical, pharmaceutical, medical, cosmetic manufacturing, paint production, polishing and fabric restoration industries (Granda, 2006).

So, the study of the extraction conditions of this product becomes of great importance and industrial interest. Thus, the objective of this study is to evaluate the effects of extraction factors such as mass-to-feed ratio (X1), particle size (X2) and time (X3), in three different levels, with their respective interactions on the crude wax yield through central compound experimental design, enabling the evaluation of best conditions for the extraction of this wax.

\section{Results and Discussion}

The data were analyzed using the Pareto diagram (Image 1) using $99 \%$ of confidence level.

Image 1: Pareto diagram of extraction factors effects on crude wax yield.

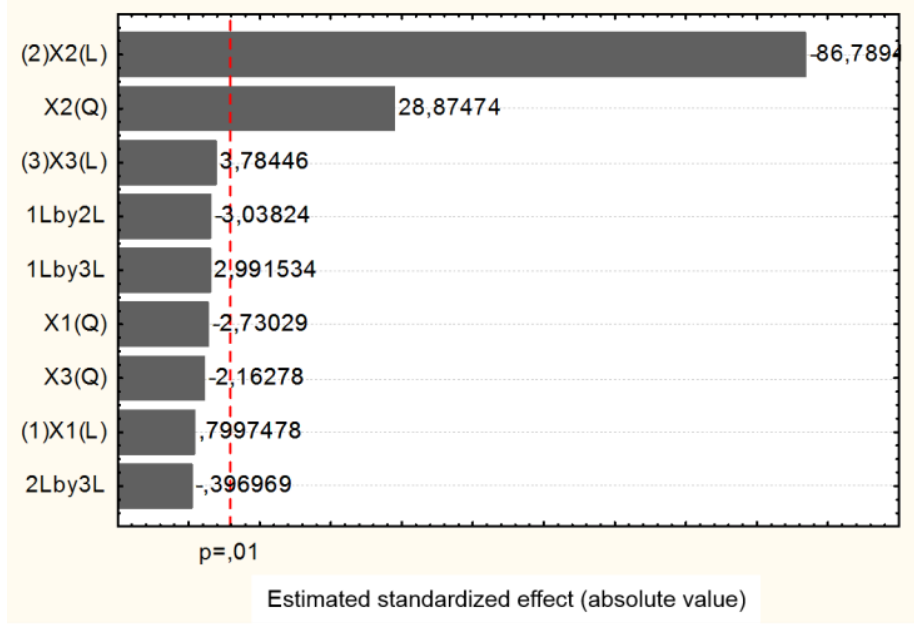

It can be inferred that considering the levels studied in this work, the only factor that influences crude wax yield extraction is the particle size, the negative sign indicates that the smaller the size, greater the yield. Probably, the phenomenon occurs due to the increase of the effectively exposed surface area of the raw material with the extractive medium, in this case, the sugarcane peel with the hexane, favoring the contact and the mass transfer between the phases. It is important to emphasize that not is possible to decrease the particle size indefinitely, since we will reach a point where it will become indivisible, also, milling and separation add costs to the process.

The interactions of the extraction factors also did not have a significant effect for the $99 \%$ confidence level and in the study range, as also observed in Image 1. Therefore, the particle size can be analyzed in isolation, as already done. Chemical properties such acid level, saponification level and unsaponifiable matter were determined and compared with commercial waxes, such Carnauba wax and Candelilla. As well as their thermal properties determined by differential scanning calorimetry (DSC) and by thermal gravimetric analysis (TGA).

\section{Conclusions}

Therefore, considering the factors levels studied in this work, it was found that is possible to extract larger amounts of wax when the particle size is decreased. It was also the only factor that proved to be significant, indicating that it can be studied independently for the optimization of the wax extraction process from sugarcane peel, without any interactions with others factors. Thus, in order to reduce process costs, one can work using less amount of solvent and less extraction time to obtain the same amount of wax.

\section{Acknowledgement}

The authors thank FAPESP (2015 / 25384-1), SAE, CAPES and CNPq for financial support.

GRANDA, K. M.; Obtenção e caracterização de cera de cana-deaçúcar e suas frações, 2006. 